\title{
LI. On the dynamical theory of electromagnetic action
}

\section{Professor Andrew Gray M.A.}

To cite this article: Professor Andrew Gray M.A. (1890) LI. On the dynamical theory of electromagnetic action, Philosophical Magazine Series 5, 30:187, 441-455, DOI: $10.1080 / 14786449008619863$

To link to this article: http://dx.doi.org/10.1080/14786449008619863

曲 Published online: 08 May 2009.

Submit your article to this journal $\lceil\pi$

Џ Article views: 2

Q View related articles $\asymp$ 
T H E

\title{
LONDON, EDINBURGH, AND DUBLIN
}

\section{PHILOSOPHICAL MAGAZINE}

\author{
AND \\ JOURNAL OF SCIENCE.
}

[FIFTH SERIES.]

$D E C E M B E R 1890$.

LI. On the Dynamical Theory of Electromagnetic Action.

By Professor Andrew Gray, M.A.*

THE use of the term potential energy in electromagnetic theory is somewhat vague. For example, an expression for the potential energy of a circuit and a magnetic shell is obtained by replacing the former by its equivalent shell and proceeding as in the magnetic theory of two shells. This expression gives the potential energy of the system in terms of the configuration of the two shells, and so enables the mutual forces between the shell and the circuit, and therefore also the work done in any relative displacement, to be calculated. But, in the presentation of the theory, the assumption is then made that this work is wholly due to the expenditure of the potential energy of the substances in the battery, and nothing is credited to a change in the intrinsic or potential energy which the system possesses irrespective of the store of energy contained in the battery.

But when we consider the case of two circuits, we have always to take into account the change in intrinsic energy. For example, when two rigid circuits are allowed to approach one another infinitely slowly, the battery, as is well known, is drawn upon for twice as much energy as is required for the work done by electromagnetic forces between the two circuits, the balance going to increase the intrinsic energy of the system.

- Communicated by the Author.

Phil. Mag. S. 5. Vol. 30. No. 187. Dec. 1890 
When the circuits are separated again by external forces applied to overcome the electromagnetic forces, the intrinsic energy is diminished by the same amount, and the battery is saved just as much energy in the reverse displacement as it supplied in the direct.

If the displacement do not take place infinitely slowly, we have to take into account the change in the intrinsic energy due to the change of the current in each circuit.

Thus the change in the intrinsic energy is here very important.

Again, when we consider two magnets which are displaced by their mutual forces, we find the change in the intrinsic energy, expressed in terms of the configuration of the system, the only thing to be taken into account. The mutual forces set the magnets into motion, or displace them against external resistance, and thus do work. This work can be obtained from no other source than the intrinsic or configuration energy of the system.

Thus in the case of two circuits and in that of two magnets, change of intrinsic energy plays a part; in the case of a magnet and a circuit it apparently does not; and I have never seen the reason of the apparent exception anywhere clearly stated. I think that the treatment of the intrinsic energy in the presentation of this part of the theory of electromagnetic action is a source of difficulty to many, and prevents clearness of ideas regarding what is certainly not found an easy subject when an attempt is made to master it in all its bearings.

This difficulty is met with in the perusal of the writings of even the greatest masters of the science of Electricity. Thus Clerk-Maxwell, in his 'Electricity and Magnetism" (vol. ii. p. 179), has given a treatment of the problem of two circuits, in which the change of intrinsic energy is left out of account, and a correct result is obtained only by what seems an inadmissible process*. This passage is a pretty close rendering of one in Helmholtz's famous essay, Die Erhaltung der Kraft in which the same treatment occurs. It is evident that here the change of intrinsic energy has not been present to the minds of the writers, and the result is a statement of theory which may have caused thoughtful students considerable embarrassment.

Again, I have observed what seem to me traces of this want of clearness in attempts to deduce directly from the expressions for the intrinsic energy of a magnetic field due to any distribution of currents, the energy dissipated per unit of volume of the medium, in carrying the substance round a closed cycle of magnetization. The correct way of

* See McConnel, 'Nature,' rol. xxxv. p. 172. 
regarding the intrinsic energy gives, as shown below, exactly the amount of energy, per unit of volume of the medium, spent in any change of magnetization, whether a complete cycle or not, otherwise than in increasing the intrinsic energy, and therefore gives at once the energy dissipated in carrying the medium through a complete cycle from one given state as to intrinsic energy back to the same state again.

Everything seems to point to the conclusion that what we are in the habit of regarding as potential energy is really kinetic energy. Every increase of scientific knowledge of matter furnishes additional proof that all its properties have their explanation in motion, and the conviction is more and more forced upon every physical student that the ordinary division of energy into potential and kinetic results from our incomplete knowledge of the material system considered. If we had perfect knowledge of the coordinates of all the parts of the system and their velocities at a given instant, and, further, knew fully the conditions to which these coordinates are subject, we should ipso facto be able to define the configuration at any time of any portion of the system, and to state how at that time the whole kinetic energy is divided between that portion and the rest of the system. Thus the ordinary transformation of potential into kinetic energy, and vice vers $\hat{a}$, is only a process of redistribution of kinetic energy between the different parts of the system.

It can in fact be proved that if a dynamical system be specified partly by a certain group of position coordinates $(\phi)$ and partly by velocities of other coordinates $(\psi)$, so that the kinetic energy is the sum of two corresponding parts $T_{\dot{\phi}}, T_{\psi}$, of which the first is expressed as a quadratic function of the velocities of the type $\dot{\phi}$, and the other as a quadratic function of the momenta corresponding to the velocities $\dot{\psi}$, the alterations of the position coordinates will take place precisely as if the system had a quantity of kinetic energy $T_{\phi}$, and a quantity of potential energy $\mathrm{T}_{\psi}{ }^{*}$. For there being no potential energy we can in this case write the Lagrangian equation for a $\phi$-coordinate as follows $\dagger$ :-

or, since $d \mathrm{~T}_{\psi} / d \dot{\phi}=0$,

$$
\frac{d}{d t} \frac{d\left(\mathrm{~T}_{\phi}-\mathrm{T}_{\psi}\right)}{d \dot{\phi}}-\frac{d\left(\mathrm{~T}_{\phi}-\mathrm{T}_{\psi}\right)}{d \dot{\phi}}=0 ;
$$

$$
\frac{d}{d t} \frac{d \mathrm{~T}_{\Phi}}{d \dot{\phi}}-\frac{d \mathrm{~T}_{\Phi}}{d \phi}=-\frac{d \mathrm{~T}_{\psi}}{d \phi}
$$

- See J. J. Thomson's 'Applications of Dynamics to Phyies and Chemistry,'p. 13.

+ Routh, 'Stability of Motion,' p. 61 ; Thomson and Tait's 'Natural Philosophy,' vol. i. part 1, p. 323 . 
which is the equation of motion we should have if $\mathrm{T}_{\psi}$ were the potential energy of the system.

Though molecular dynamics, notwithstanding its great advances, may never succeed in giving us a perfect knowledge of the coordinates, which are thus ignored in the sense that the energy is expressed without them, still in some cases, as in the general dynamical treatment of electric currents introduced by Maxwell, it adds greatly to clearness of statement and view to adopt the idea that the intrinsic energy of the system, or that part of it alone concerned in electromagnetic action, is really kinetic energy. Thus, in using the expression for this electrokinetic energy in the Lagrangian equations, we find the electromotive forces of induction, and the electromagnetic forces between the parts of the system.

It has been shown by Maxwell (Electricity and Magnetism, vol. ii. chap. vi.), and the proof need not be repeated here, that the electrokinetic energy of a system of current-carrying conductors may be written as a homogeneous quadratic function of the current-strengths, which are regarded as velocities corresponding to generalized coordinates $y_{1}, y_{2}$, and specifying the different circuits. Adopting the notation $\dot{y}_{1}, \dot{y}_{2}$, \&c. for these velocities, and putting $\mathrm{T}$ for the electrokinetic energy, we have

$$
\mathrm{T}=\frac{1}{2}\left(\mathrm{~L}_{1} \dot{y}_{1}{ }^{2}+2 \mathrm{M}_{12} \dot{y}_{1} \dot{y}_{2}+\ldots+\mathrm{L}_{2} \dot{y}_{2}{ }^{2}+2 \mathrm{M}_{23} \dot{y}_{2} \dot{y}_{3}+\ldots\right) ; .
$$

where $\mathrm{L}_{1}, \mathrm{M}_{12}$, \&c., $\mathrm{L}_{2}, \mathrm{M}_{23}$, \&c. are coefficients involving only the ordinary position coordinates of the material system, and fulfilling the relation $M_{p q}=M_{q p} . L_{1}, L_{2}$, \&c. are in fact the coefficients of self-induction for the different circuits $\mathrm{M}_{12}, \mathrm{M}_{13}$, and $\mathrm{M}_{23}$ \&c. the coefficients of mutual induction for the pairs of circuits indicated by the suffixes.

If we denote the magnetic inductions through the different circuits by $\mathrm{N}_{1}, \mathrm{~N}_{2}, \ldots \mathrm{N}_{k}, \ldots$, we have

$$
\begin{aligned}
& \mathrm{N}_{1}=\frac{d \mathrm{~T}}{d \dot{y}_{1}}=\mathrm{L}_{1} \dot{y}_{1}+\mathrm{M}_{12} \dot{y}_{2}+\ldots+\mathrm{M}_{1 k} \dot{y}_{k}+\ldots, \text {, } \\
& \left.\mathrm{N}_{2}=\frac{d \mathrm{~T}}{d \dot{y}_{2}}=\mathrm{M}_{21} \dot{y}_{1}+\mathrm{L}_{2} \dot{y}_{2}+\ldots+\mathrm{M}_{2 k} \dot{y}_{k}+\ldots,\right\} \text {. } \\
& \mathrm{N}_{k}=\frac{d \mathrm{~T}}{d \dot{y}_{k}}=\mathrm{M}_{k 1} \dot{y}_{1}+\mathrm{M}_{k 2} \dot{y}_{2}+\ldots+\mathrm{L}_{k} \dot{y}_{k}+\ldots ;
\end{aligned}
$$

so that (1) becomes

$$
\mathrm{T}=\frac{1}{2}\left(\mathrm{~N}_{1} \dot{y}_{1}+\mathrm{N}_{2} \dot{y}_{2}+\ldots+\mathrm{N}_{k} \dot{y}_{k}+\ldots\right) . . . . .
$$


The quantities $\mathrm{N}_{1}, \mathrm{~N}_{2}$, \&c. are generalized components of electrokinetic momentum.

Using Lagrange's equations of motion and introducing Lord Rayleigh's Dissipation Function F, we hare, since the coefficients in $\mathrm{T}$ do not contain the coordinates $y$, for the impressed electromotive forces in the different circuits the typical equation

But

$$
\frac{d \mathrm{~N}_{k}}{d t}+\frac{d \mathrm{~F}}{d \dot{y}_{k}}=\mathrm{E}_{k} . . . . . . .
$$

$$
\mathrm{F}=\frac{1}{2} \sum \mathrm{R}_{k} \dot{y}_{k}^{2}
$$

where $R_{k}$ is the resistance in the circuit indicated by the suffix $k$. Hence

$$
\frac{d \mathrm{~N}_{k}}{d t}=\mathrm{E}_{k}-\mathrm{R}_{k} \dot{y}_{k} . . . . \quad . \quad .
$$

The electromotive forces of the type $d \mathrm{~N}_{k} / d t$ are the parts of the impressed electromotive forces which are employed in working against the electromotive forces due to induction. Thus $-d \mathbf{N}_{k} / d t$ is the type of the actual electromotive force due to induction. Since this may be written in the form

$$
-\frac{d}{d t} \frac{d \mathrm{~T}}{d \dot{y}_{k}}
$$

we see that the inductive electromotive force tends to diminish the electrokinetic energy.

Again, the forces which must be applied to work against the reacting internal electromagnetic forces are

$$
-\frac{d \mathrm{~T}}{d x_{1}}, \quad-\frac{d \mathrm{~T}}{d x_{2}}, \& c .
$$

where $x_{1}, x_{2}$, \&c. are coordinates determining the geometrical positions of the circuits. Thus, the mutual electromagnetic forces having equal and opposite values to these, tend to increase the electrokinetic energy. These two results must be kept clearly in mind in what follows.

This dynamical theory, if applicable to a system of ordinary circuits, must, if Ampère's theory of magnetism be true, be also applicable to a system of magnets, or a system composed partly of ordinary circuits and partly of magnets ; otherwise Ampère's theory cannot be a complete expression of the facts of the case. Maxwell has applied it only to the case of two circuits, and its applicability to the case of a circuit and a 
magnetic shell has been questioned by Mr. S. H. Burbury (Proc. Camb. Phil. Soc. 1888). I shall endeavour to test its applicability by a comparison of its consequences in the three simple typical cases :-(1) two ordinary circuits, (2) an ordiuary circuit and a magnetic shell, (3) two magnetic shells.

(1) In this case let $L_{1}, L_{2}, M$ be the coefficients of selfinduction, and the coefficient of mutual induction for the two circuits. Then we have

$$
\mathrm{T}=\frac{1}{2}\left(\mathrm{~L}_{1} \dot{y}_{1}{ }^{2}+2 \mathrm{M} \dot{y}_{1} \dot{y}_{2}+\mathrm{L}_{2} \dot{y}_{2}{ }^{2}\right) . \quad . \quad . \quad .
$$

If $E_{1}, E_{2}$ be the electromagnetic forces, $R_{1}, R_{2}$ the resistances, equation (5) gives

$$
\left.\begin{array}{l}
\mathrm{E}_{1}-\frac{d}{d t}\left(\mathrm{~L}_{1} \dot{y}_{1}+\mathrm{M} \dot{y}_{2}\right)=\mathrm{R}_{1} \dot{y}_{1}, \\
\mathrm{E}_{2}-\frac{d}{d t}\left(\mathrm{~L}_{2} \dot{y}_{2}+\mathrm{M} \dot{y}_{1}\right)=\mathrm{R}_{2} \dot{y}_{2}
\end{array}\right\} . . . .
$$

We shall suppose that the circuits are rigid, so that $L_{1}, L_{2}$ are invariable. Then if the circuits be subjected only to those changes which take place from their mutual action, and $d \mathrm{~T}$ be the change in $T$ which takes place in a small interval of time $d t$,

$$
d \mathrm{~T}=\mathrm{L}_{1} \dot{y}_{1} d \dot{y}_{1}+\mathrm{M}\left(\dot{y}_{2} d \dot{y}_{1}+\dot{y}_{1} d \dot{y}_{2}\right)+\mathrm{L}_{2} \dot{y}_{2} d \dot{y}_{2}+\dot{y}_{1} \dot{y}_{2} d \mathrm{M} .
$$

The work $d \mathrm{~W}$ done by electromagnetic forces has the value $d \mathrm{~T} / d x \cdot d x$. Hence

$$
d \mathrm{~W}=\dot{y}_{1} \dot{y}_{2} d \mathrm{M} . \quad . \quad . \quad . \quad .
$$

This work is spent in producing kinetic energy in the displaced conductors (or if these are not free, in moving them against external resistance, or in both ways).

The work done by the impressed electromotive forces over and above that dissipated is

$$
\left(\mathrm{E}_{1}-\frac{d \mathrm{~F}}{d \dot{y}_{1}}\right) \dot{y}_{1} d t+\left(\mathrm{E}_{2}-\frac{d \mathrm{~F}}{d \dot{y}_{2}}\right) \dot{y}_{2} d t,
$$

which, by (7), has the value

$$
\mathrm{I}_{1} \dot{y}_{1} d \dot{y}_{1}+\mathrm{M}\left(\dot{y}_{1} d \dot{y}_{2}+\dot{y}_{2} d \dot{y}_{1}\right)+\mathrm{I}_{2} \dot{y}_{2} d \dot{y}_{2}+2 \dot{y}_{1} \dot{y}_{2} d \mathrm{M},
$$

or, by (8) and (9), the value $d \mathrm{~T}+d \mathrm{~W}$, so that the energy is all accounted for.

This agreement (as also that in each of the cases which follow) is of course only a consequence of the equations given above; but its exhibition tends to give a clearer notion of the dynamical meaning of these equations. 
Here part of the impressed electromotive forces $\mathrm{E}_{1}, \mathrm{E}_{2}$ in each case is employed in working against the inductive electromotive force, and thus in increasing the electrokinetic energy. By the electromagnetic forces the electrokinetic energy is increased by the amount $\dot{y}_{1} \dot{y}_{2} d \mathrm{M}$, which also is the amount of work spent in moving the circuits.

If the conductors are displaced from rest to rest again, so that $\dot{y}_{1}, \dot{y}_{2}$, have resumed their steady values $d \dot{y}_{1}=0, d \dot{y}_{2}=0$, the energy furnished by the batteries is $2 \dot{y}_{1} \dot{y}_{2} d \mathrm{M}$, of which one half is accounted for in $d \mathrm{~T}$, the other by the work done in moving the circuits, which has its equivalent in work done against the external resistance by which the conductors were brought to rest. This result was obtained by Sir William Thomson so long ago as 1851 .

(2) Assuming, according to Ampère, that a magnetic shell is equivalent to a current round its edge, and putting $\dot{y}_{2}$ for this current, we have, since $\mathrm{E}_{2}=0, \mathrm{R}_{2}=0$, instead of (7),

$$
\left.\begin{array}{rl}
\mathrm{E}_{1}-\frac{d}{d t}\left(\mathrm{~L}_{1} \dot{y}_{1}+\mathrm{M} \dot{y}_{2}\right) & =\mathrm{R}_{1} \dot{y}_{1} \\
d & \left.d \mathrm{~L}_{2} \dot{y}_{2}+\mathrm{M} \dot{y}_{1}\right)=0 .
\end{array}\right\}
$$

As before we get

$$
\begin{aligned}
d \mathrm{~T} & =\mathrm{L} \dot{y}_{1} d \dot{y}_{1}+\mathrm{M}\left(\dot{y}_{2} d \dot{y}_{1}+\dot{y}_{1} d \dot{y}_{2}\right)+\mathrm{L}_{2} \dot{y}_{2} d \dot{y}_{2}+\dot{y}_{1} \dot{y}_{2} d \mathrm{M}, \\
d \mathrm{~W} & =\dot{y}_{1} \dot{y}_{2} d \mathrm{M} .
\end{aligned} .
$$

The energy furnished by the battery is, as before,

$$
\mathrm{L}_{1} \dot{y}_{1} d \dot{y}_{1}+\mathrm{M} \dot{y}_{1} d \dot{y}_{2}+\dot{y}_{1} \dot{y}_{2} d \mathrm{M} \text {. }
$$

By the other circuit no energy is given. But multiplied by $\dot{y}_{2}$ the second of $(10)$ gives

$$
\mathrm{L}_{2} \dot{y}_{2} d \dot{y}_{2}+\mathrm{M} \dot{y}_{2} d \dot{y}_{1}+\dot{y}_{1} \dot{y}_{2} d \mathrm{M}=0 \text {. }
$$

Thus

$$
\left.\begin{array}{rl}
d \mathrm{~T} & =\mathrm{L} \dot{y}_{1} d \dot{y}_{1}+\mathrm{M} \dot{y}_{1} d \dot{y}_{2} \\
d \mathrm{~T}+d \mathrm{~W} & =\mathrm{L}_{1} \dot{y}_{1} d \dot{y}_{1}+\mathrm{M} \dot{y}_{1} d \dot{y}_{2}+\dot{y}_{1} \dot{y}_{2} d \mathrm{M},
\end{array}\right\}
$$

which again, of course, is the energy furnished by the battery.

Here, if the changes are estimated for the system when brought to rest, $d \dot{y}_{1}=0$. The other current $\dot{y}_{2}$ does not, however, remain constant, and we have to inquire what is its effect upon the magnet. We know that the moment of a hard magnet is not seriously altered by a displacement produced by the mutual forces if $\dot{y}_{1}$ is moderate; and the small alteration 
generally observed is opposite to that which would be produced by the inductive change in $\dot{y}_{2}$. We are led to conclude that $\dot{y}_{2}$, on which, according to Ampère's theory, the intensity of magnetization depends, must remain practically constant. Writing, then, the second of $(10)$ in the form

$$
\mathrm{L}_{2} \dot{y}_{2}+\mathrm{M} \dot{y}_{1}=\mathrm{L}_{2} \gamma \text {, }
$$

where $\gamma$ is the initial current before the magnet was brought into the field of the circuit, we see that if the dynamical theory is applicable, $\mathrm{M} \dot{y}_{1} / \mathrm{L}_{2}$ must be a quantity small in comparison with $\gamma$. This gives $d \dot{y}_{2}$ a small quantity of the second order, and makes the value of $d^{\prime} \mathrm{l}$, the change in the electrokinetic energy, depend only on the term $L_{1} \dot{y}_{1} d \dot{y}_{1}$, which is zero if the system is not in relative motion. 'Thus the fact that the mechanical value of a current in a conductor is not affected by bringing permanent magnets into its neighbourhood is not contradicted by the dynamical theory, if the supposition hero made as to the value of $\mathrm{M} \dot{y}_{1} / \mathrm{L}_{2}$ be actually true. We hare to inquire what jhysical reasons can be adduced in stipport of it.

Suppose that, instead of a simple magnetic shell, we have a solenoid made up of equal distinct circuits, in each of which a current $\gamma$ flows. If the number of circuits per unit of length be $n$, the coefficient of self-induction per unit of length is $4 \pi n^{2} \mathrm{~A}$, where $\mathrm{A}$ is the area of the circuit, and the total induction through the solenoid (neglecting the effect of its ends) is $4 \pi n^{2} l \gamma \AA$, if $l$ be its lengtb. If the circuit carrying the current $\dot{y}_{1}$ consist of $n^{\prime}$ turns of wire closely surrounding the sulenoid, the induction through it and the solenoid is $4 \pi n n^{\prime} \gamma \mathrm{A}$. Thus the maximum value of $\mathrm{M}$ is $4 \pi n n^{\prime} \mathrm{A}$. Thus we get

$$
\frac{\mathrm{M}}{\mathrm{L}_{2}}=\frac{n^{\prime}}{n l} \text {; }
$$

that is, the ratio, which the theory indicates must be vanishingly small, is equal to the ratio of the number of turns in the circuit to the number of circuits in the solenoid. Thus we are led to the supposition, probable on other grounds, that the number of molecular circuits in the solenoid is exceedingly great.

It is also necessary, in order that the Amperian molecules may give an inductive magnetization in iron agreeing with experiment, that the self-induction of each molecular circuit must be great, that is $A / L_{2}$ must be small. If the current flow in a ring channel this condition; as Maxwell has pointed out*, may be fulfilled by supposing the radius $R$ of the mean

* Maxwell, 'Elcctricity and Magnetism,' vol. ii. $\{844$. 
line of the channel great in comparison with $r$ the radius of the channel, since $\mathrm{L}_{2}$ depends on $\log \mathrm{R} / r$.

(3) Considering now the case of two shells, we have the same expressions for $d \mathrm{~T}$ and $d \mathrm{~W}$ as in $\mathrm{in}_{\mathrm{N}}(11)$; but the equations (10) become, since now $R_{1}=0, E_{1}=0$,

$$
\left.\begin{array}{l}
\mathrm{L}_{1} \dot{y}_{1} d \dot{y}_{1}+\mathrm{M} \dot{y}_{1} d \dot{y}_{2}+\dot{y}_{1} \dot{y}_{2} d \mathrm{M}=0 \\
\mathrm{~L}_{2} \dot{y}_{2} d \dot{y}_{2}+\mathrm{M} \dot{y}_{2} d \dot{y}_{1}+\dot{y}_{1} \dot{y}_{2} d \mathrm{M}=0 .
\end{array}\right\}
$$

Thus substituting in (11) we find

$$
d \mathrm{~T}=-\dot{y}_{1} \dot{y}_{2} d \mathrm{M}, .
$$

which is equal and opposite to the work done by the electromagnetic forces in moving the system. Here, it is to be observed, the electrokinetic energy is diminished through the action of the electromotive forces by just double the amount by which the electromagnetic forces increase it.

The performance of a finite amount of work by electromagnetic forces in overcoming external forces, or in giving the magnets kinetic energy, and the diminution of the electrokinetic energy by an equal amount, are quite consistent with only a slight alteration of $\dot{y}_{1}, \dot{y}_{2}$, and therefore of the magnetic moments of the magnets. Indeed, the alteration in the magnetic moments of the magnets actually produced is opposite in sign to that which would be caused by $d \dot{y}_{1}, d \dot{y}_{2}$, and is due no doubt to magnetization produced by a turning round of magnetic molecules in the body. But in an ideal case, in which the molecules could undergo no such alteration of position, it is only necessary to suppose that $\mathrm{L}_{1}, \mathrm{~L}_{2}$ are each great in comparison with $M$ to enable the effect of $d \dot{y}_{1}, d \dot{y}_{2}$ on the magnetic moments to be small, a supposition which is very possibly in accordance with fact.

There does not seein to me, therefore, to be any insuperable physical difficulty in the way of the application of the general dynamical equations to magnets as well as to circuits carrying currents. Ot course our ignorance of the current strength, $\& c .$, in the former case prevents us from making practical use of these equations; but their theoretical application gives I think a clearer view of the energetics of electromagnetic (and magnetic) action than can be obtained without them.

I shall now consider from this dynamical point of view the inductive magnetization produced in iron and other substances when placed in a magnetic field. As is well known, the expression in (1) or (3) for the electrokinetic energy can be reduced to a definite integral. The following method of performing this reduction seems more direct and simple than 
that used by Maxwell, which is the method I think generally pursued. Starting from equation (3), viz.,

$$
\mathrm{T}=\frac{1}{2}\left(\mathrm{~N}_{1} \dot{y}_{1}+\mathrm{N}_{2} \dot{y}_{2}+\ldots+\mathrm{N}_{k} \dot{y}_{k}+\ldots\right),
$$

take any circuit, say that through which the induction is $\mathrm{N}_{k}$, and draw any surface in the field so as to form a cap with that circuit as bounding edge. Then, by the well-known theorem as to the work done in carrying a unit pole in a closed path round a circuit, we have

$$
4 \pi \dot{y}_{k}=\int \mathrm{H}_{k} \cos \theta_{k} d s,
$$

where the integration is taken round any closed path embracing the circuit, $\mathrm{H}_{k}$ is the magnetic force due to the current in that circuit, and $\theta_{k}$ the angle which $\mathrm{H}_{k}$ makes with $d s$, an element of the path. Hence, since the total induction through every such surface is the same, we have

$$
\mathrm{N}_{k} \dot{y}_{k}=\frac{1}{4 \pi} \int \mathrm{N}_{k} \mathrm{H}_{k} \cos \theta_{k} d s . \quad . \quad . \quad .
$$

Now let the surface be taken at right angles to the lines of induction everywhere. These lines are closed curves round the conductors, and each threads throngh one or more of the circuits. It is possible to divide up the whole field by successive surfaces, each having for bounding edge any given circuit, so that every one of those surfaces shall be everywhere at right angles to the lines of induction. Every one of these, if it cut through a system of closed lines belonging to any one circuit, will pass through every point of that circuit. Of course no one of the closed tubes of induction which the surface thus cuts through contributes anything to the total induction through the surface.

Now let the direction of the closed curve, round which the integral of $\mathrm{H} \cos \theta d s$ is taken, be everywhere at right angles to these surfaces, and let $B$ be the value of the induction at any point where this curve cuts one of these surfaces. Then, if $d S$ be the area of an element of the surface at that point, the induction through it is $\mathrm{B} d \mathrm{~S}$. Thus we get

$$
\mathrm{N}_{k} \dot{y}_{k}=\frac{1}{4 \pi} \iint \mathrm{BH}_{k} \cos \theta_{k} d s d \mathrm{~S},
$$

where one integral is taken over the surface and the other round the closed curre. But this is evidently the same thing as 


$$
\mathrm{N}_{k} \dot{y}_{k}=\frac{1}{4 \pi} \iint_{-\infty}^{+\infty} \int_{-\infty} \mathrm{BH}_{k} \cos \theta_{k} d x d y d z,
$$

where $x, y, z$ are the coordinates of the point at which the induction is $\mathrm{B}$, and $d x d y d z$ is an element of volume.

Finally, take any point on one of these surfaces, and let $B$ be the induction there. For every such point a surface can be drawn having any one of the circuits as its boundary, and hence by (1.6) and (3) we bave finally for $\mathrm{T}$ the equation:

$$
\mathrm{T}=\frac{1}{8 \pi} \iint_{-\infty}^{+\infty} \int_{-\infty} \mathrm{B}\left(\mathrm{H}_{1} \cos \theta_{1}+\mathrm{H}_{2} \cos \theta_{2}+\& \mathrm{c} .\right) d x d y d z .
$$

But if $\mathrm{H}$ be the total magnetic force at the point and $\theta$ the angle which it makes with the normal to the surface, we have

$$
\mathrm{H} \cos \theta=\mathrm{H}_{1} \cos \theta_{1}+\mathrm{H}_{2} \cos \theta_{2}+\& \mathrm{c} \text {. }
$$

Hence (17) becomes

$$
\mathrm{T}=\frac{1}{8 \pi} \iiint \mathrm{BH} \cos \theta d x d y d z . \quad . \quad .
$$

If we suppose, what is always the case in an isotropic medium, that $\mathrm{B}$ and $\mathrm{H}$ have the same direction, we have

$$
\mathrm{T}=\frac{1}{8 \pi} \iint_{-\infty}^{+\infty} \int_{-\infty} \mathrm{BH} d x d y d z=\frac{1}{8 \pi \mu} \iint_{-\infty}^{+\infty} \int_{\mathrm{B}^{2}} d x d y d z,
$$

since $\mathrm{B}=\mu \mathrm{H}$, if $\mu$ be the magnetic inductive capacity or per. meability of the medium.

Now let the total induction through the circuit in which the magnetizing current is flowing be increased by an amount $d \mathrm{~N}$ produced by increasing the current in that circuit. The energy drawn from the battery is $\dot{y} d \mathrm{~N}$; and clearly by the investigation given above, we have, if $d \mathrm{~B}$ be the increase of induction at $(x, y, z)$,

$$
\dot{y} d \mathrm{~N}=\frac{1}{4 \pi} \iint_{-\infty}^{+\infty} \int_{-} \mathrm{H} d \mathrm{~B} d x d y d z . \quad . .
$$

The change of electrokinetic energy $d \mathrm{~T}$ is given by

$$
d \mathrm{~T}=\frac{1}{8 \pi} \iint_{-\infty}^{+\infty} \int(\mathrm{H} d \mathrm{~B}+\mathrm{B} d \mathrm{H}) d x d y d z . \quad .
$$


Hence for the energy spent otherwise than in increasing the electrokinetic energy in the medium, we have

$$
\dot{y} d \mathrm{~N}-d \mathrm{~T}=\frac{1}{4 \pi} \int_{-\infty}^{+\infty} \iint_{-\infty}\left\{\mathrm{H} d \mathrm{~B}-\frac{1}{2}(\mathrm{H} d \mathrm{~B}+\mathrm{B} d \mathrm{H})\right\} d x d y d z .
$$

If the magnetization be carried through a closed cycle, so that the medium is brought back to the same state as at first, the electrokinetic energy returns to the same value, and the integral of the quantity within the inner brackets, which is $d(\mathrm{BH})$, is zero. Thus the energy furnished to the medium in the closed cycle is

$$
\frac{1}{4 \pi} \iint_{-\infty}^{+\infty} \int\left\{\int \mathrm{H} d \mathrm{~B}\right\} d x d y d z
$$

the inner integral being taken with respect to $B$ round the cycle.

If we take the changes per unit of volume at a place where the induction is $\mathrm{B}$ and the magnetic force $\mathrm{H}$, we have for the energy given to the medium the value $\mathrm{H} d \mathrm{~B} / 4 \pi$, and for the increase of electrokinetic energy $d(\mathrm{BH}) / 8 \pi$. Therefore, for the energy spent otherwise than in increasing the electrokinetic energy, we get the expression

$$
\frac{1}{4 \pi} \mathrm{H} d \mathrm{~B}-\frac{1}{8 \pi}(\mathrm{H} d \mathrm{~B}+\mathrm{B} d \mathrm{H}) \text {. }
$$

Hence if $\mathrm{P}, \mathrm{Q}$ (fig. 1) be two points on a curve of magnetization of which the ordinates are values of $B$, and the

Fig. 1.

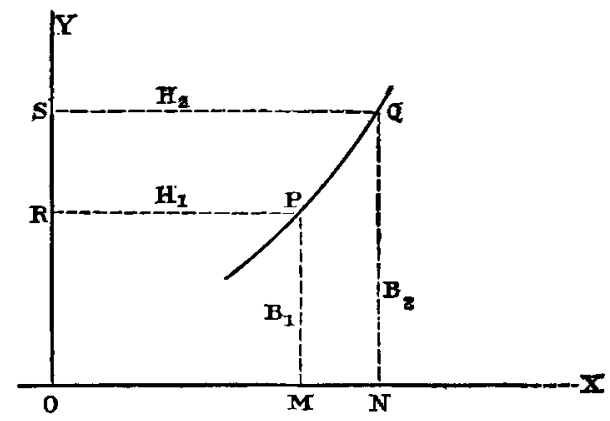

abscissæ values of $H$, we have for the whole energy spent otherwise than in increasing $\mathrm{T}$, in this part of the curve, the value 
that is,

$$
\frac{1}{4 \pi}\left\{\int_{B_{1}}^{B_{2}} \mathrm{H} d \mathrm{~B}-\frac{1}{2} \int_{B_{1}}^{B_{2}}(\mathrm{H} d \mathrm{~B}+\mathrm{B} d \mathrm{H})\right\} \text {; }
$$

$$
\frac{1}{4 \pi}\left(\text { area } P \text { Q S R }-\frac{1}{2}\right. \text { area N QS R PM). }
$$

The second area of course vanishes when $Q$ coincides with $P$, as it does when the curve forms a closed cycle.

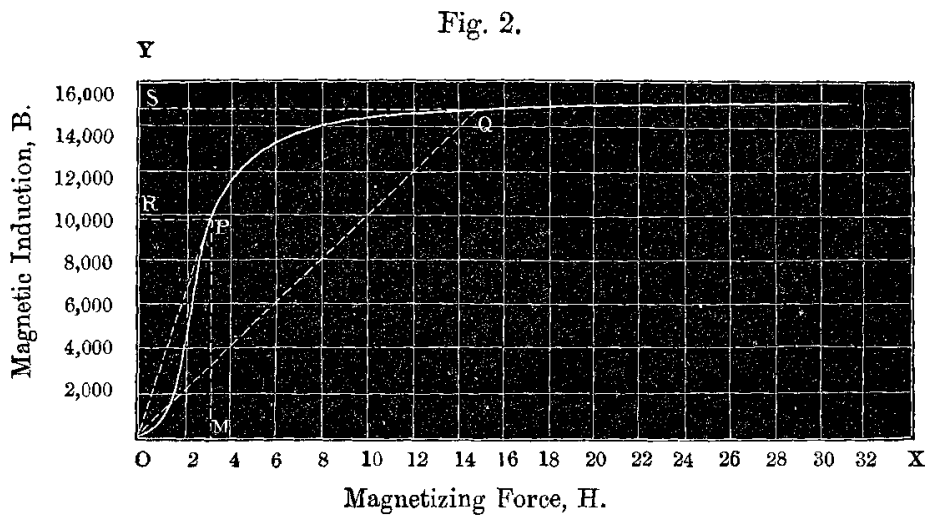

It is interesting to apply this result to an actual curve of magnetization in iron (fig. 2). For all points on the curve up to a little distance beyond $\mathrm{P}$, the total energy given to the medium in producing the corresponding magnetization exceeds the electrokinetic energy, but for all points further from the origin the electrokinetic energy exceeds, and for points on the upper flat part of the curve very greatly exceeds, the energy given out to the medium by the battery. [The point $P$ at which $O P$ is a tangent to the curve marks the point at which the energy given out to the medium and the electrokinetic energy are increasing at the same rate, the former below that point is increasing faster, above that point slower than the latter.]

We are forced to conclude that for every series of magnetization steps from zero up to a certain point energy is given to the medium, and for every series from zero up to any further point the medium furnishes the balance of energy required for the electrokinetic energy. In every small step below $\mathrm{P}$ a balance of energy, over and above the electrokinetic energy, is given to the medium; for every small step 
abore $\mathrm{P}$ a balance is taken from the medium to make up the electrokinetic energy.

It seems to me that these results are very intimately connected with and confirm Prof. Ewing's theory of Magnetization. published in the Philosophical Magazine for September. In the steep part of the curve beyond the first bend we bave work done by the electromagnetic forces (and probably converted into heat) at a much greater rate than elsewhere in the curve. Here, according to this theory, magnets undergo almost all the turning of their axes they receive. Hence the coefficients of mutual induction must increase here most quickly, and consequently also the work done by electromagnetic forces which depend, as shown above, on the changes in these coefficients.

Prof. Ewing supposes that the molecular magnets having been swung round into new positions of stable equilibrium vibrate about that position, generating Foucault currents in the surrounding medium, thus heating it. Now Baur, Rowland, Hopkinson, and others have shown that iron has its magnetic susceptibility increased by rise of temperature so long as a certain high magnetizing force and a certain high temperature are not exceeded. The conclusion which follows from this by thermodynamic theory is that increase of magnetization should cool the iron. Although of course we cannot reason rigidly for an unclosed series of changes, owing to our want of knowledge of the molecular changes, it does seem probable that the iron is really beated in the part of the curve for which the curvilinear area $O Q S$ is greater than the triangle $\mathrm{OQS}$. The hypothesis would, however, explain this seeming contradiction; for, as I understand it, the thermodynamic theory does not take into account any expenditure in producing heat of the electromagnetic work done in altering the magnetic state. If the molecules could be laid hold of by external forces, against which they might do work in going round to their new positions, so as to be guided to rest in these positions, there would be no vibration, and the iron would show a cooling, according to thermodynamic theory. In fact the heating due to the Foucault currents, supposed to be thus generated, must be less than it otherwise would be by the thermodynamic cooling.

In the upper part of the curve it seems probable that there is a marked cooling. At present I am engaged in a careful experimental investigation of heating-effects for the whole curve.

Another point which may be here remarked is that (as 
shown in equations (13) for a particular case) the inductions through the molecular circuits remain constant. Hence in a magnetizable body these inductions must be the same in the unmagnetized state of the body as in its magnetized state; and this seems to show that the magnetization consists in the turning round, into facing on the whole in the same direction, different lines or chains of regularly arranged molecular magnets, or molecular circuits, as opposed to an alignment of a number of circuits previously perfectly mixed up in their arrangement. For if each of these chains have its component molecules all facing the same way, and the different chains be so arranged that the body has no magnetic moment in mass, the constant induction of large amount through each circuit can be accounted for ; and this appears to me to be the more natural explanation. A body which had its molecular circuits perfectly mixed up as to direction could not, it seems to me, be magnetized at all, except in so far as to produce an induction through each of the circuits equal and opposite to the integral of the magnetizing force over that circuit, unless indeed the coefficients of self-induction are in all cases so large as to give a considerable induction through each circuit due to its own current alone. If the latter were the case, the result of causing all the molecular circuits to face one way would be to produce an induction, in the same direction, over every part of a section of the body across the direction of the magnetizing force; if it were not the case, the induction across such a section would be everywhere small, in consequence of the fact that the surrounding circuits before the alignment contribute on the whole nothing to the induction through a particular circuit, which induction must afterwards still have the same small value.

Thus, if the current in each circuit does not by itself produce an induction in itself considerable, a moderate magnetizing force, applied to a body composed of molecules perfectly mixed as to the directions of their axes, ought to produce diamagnetic quality, in consequence of the creation of the magnetic induction required to keep the total induction through each circuit at its former value.

Of course diamagnetic quality is most simply accounted for by supposing the molecules immovable. The only effect of placing the body in a magnetic field would then be to produce induced currents corresponding to diamagnetization. 